\title{
TTR
}

Traduction, terminologie, re?daction

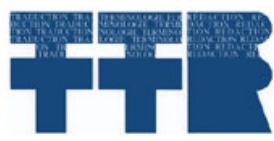

\section{Éprouver le passage}

\section{Monique Schneider}

Volume 11, numéro 2, 2e semestre 1998

Psychanalyse et traduction : voies de traverse

Psychoanalysis and Translation: Passages Between and Beyond

URI : https://id.erudit.org/iderudit/037334ar

DOI : https://doi.org/10.7202/037334ar

Aller au sommaire du numéro

\section{Éditeur(s)}

Association canadienne de traductologie

ISSN

0835-8443 (imprimé)

1708-2188 (numérique)

Découvrir la revue

Citer cet article

Schneider, M. (1998). Éprouver le passage. TTR, 11(2), 55-72.

https://doi.org/10.7202/037334ar

\section{Résumé de l'article}

Éprouver le passage - Lorsque se trouve accentuée la dimension de perte inhérente à l'accès au symbolique, la traduction peut apparaître comme le champ privilégié où s'étale cette perte. Direction suivie par J.-B. Pontalis parlant de « la mélancolie du langage » et dotant le traducteur de « la capacité infinie d'être triste ». Une autre orientation se dessine si la traduction est envisagée dans son exercice même et non dans son seul résultat. L'expérience analysée sera celle de la retraduction des textes de Freud, mettant au jour les opérations à l'oeuvre dans les traductions officielles : maquillage des termes se rapportant à la scène de séduction et à la possibilité, pour le maître, d'avoir accès à la jouissance. Ce qui conduit à une mise en abyme du procès de la séduction.

De la traduction mystifiée à la traduction impossible : c'est la tentative même de franchissement qui fait apparaître l'inconvertible dans une langue, l'exemple choisi étant le signifiant allemand, Leib, terme désignant, par opposition à Körper, le corps vivant ou le corps en creux, donc connoté de féminité; connotation qu'aucun terme français ne pourra rendre.

Tous droits réservés ( $\odot$ TTR: traduction, terminologie, rédaction — Les auteurs, Ce document est protégé par la loi sur le droit d’auteur. L’utilisation des 1998 services d'Érudit (y compris la reproduction) est assujettie à sa politique d'utilisation que vous pouvez consulter en ligne.

https://apropos.erudit.org/fr/usagers/politique-dutilisation/ 


\section{Éprouver le passage}

\section{Monique Schneider}

Si la perte censée accompagner toute entreprise de traduction a régulièrement fait l'objet de propos élégiaques - on ne saurait traduire sans trahir -, cette même perte n'est-elle pas susceptible, aujourd'hui, de s'insérer dans un processus de célébration, à partir du moment où l'on voit en elle l'épreuve marquant le passage de l'illusion à la lucidité désenchantée? Toute l'orchestration psychanalytique du * travail de deuil * conduit à focaliser l'attention sur la traversée de quelque déception initiatique, donnant accès à une pensée délestée de tout mirage. Or dans Perdre de vue, J.-B. Pontalis voit dans le travail de traduction l'un des champs privilégiés où se réaliserait cet apprentissage d'une épreuve dégrisante, forme moderne de la discipline du renoncement.

\section{L'impératif mélancolique}

Dans la mesure où la fonction initiatique dévolue à l'expérience de perte fait l'objet d'un acte de foi, n'accède-t-elle pas, dans la théorie psychanalytique, à un statut quelque peu mythique? La psychanalyse n'est pas sans entretenir un rapport ambigu avec le champ mythique, ambiguité qui trouve à se lire dans le rôle conféré au mythe œdipien. En tant qu'il soutient l'une des hypothèses fondatrices de la psychanalyse, le mythe constitue certes l'équivalent d'une matrice. Toutefois, le geste œdipien prenant, aux yeux de Freud, une valeur paradigmatique - la solution de l'énigme provoquant la chute de la Sphinx - met en scène l'efficience dissolvante d'une * solution * qui réduit d'emblée l'opacité apparente de l'énigme, rendant ainsi inutile le recours à un langage crypté. En 
proposant de répondre "l'homme " là où se déployait une série de figures campant des âges successifs et hétérogènes, Edipe ne se livre-t-il pas à une traduction magistrale, se voulant sans restes? D'où la chute logique de la Sphinx. Freud reprendra d̀ son compte cet idéal interprétatif dans son rêve de la "solution " injectée à Irma, le terme disant la " solution " (Lösung) pouvant également être traduit par * dissolution *. Ce qui est appelé à se dissoudre n'a-t-il pas trait au mode d'énonciation de l'énigme, énigme inséparable du chant qui la module? L'écoute de la parole peutelle reposer sur la neutralisation préalable des modulations émises par * cette chienne qui nous ensorcelait avec ses chants $\approx$, pour recourir aux mots de Sophocle? Cette question s'est imposée lors d'une investigation sur le statut de la parole dans le jeu analytique (Schneider, 1980); elle rebondit dans cette interrogation sur ce qu'on pourrait nommer le mythe freudien, mythe reposant sur la fiction d'une traduction dissolvante. Par le congé qu'il semble donner à la parole chantée, le mythe œdipien ne tend-il pas à sonner le glas de l'ère mythique, dans la mesure où il procède à la dissolution d'un langage par énigmes? Dans * Une difficulté de la psychanalyse ", Freud insérera d'ailleurs la leçon psychanalytique dans une série de verdicts ayant pour effet d'imposer une * humiliation $"$ à des illusions soutenues par le narcissisme, comme si les progrès des Lumières ne pouvaient se réaliser qu'en ruinant un discours qui serait animé, porté par le rêve et l'illusion qui en sont solidaires.

J.-B. Pontalis s'inscrit dans cet héritage déceptif en plaçant l'accès au langage et l'engagement dans l'analyse, non sous le signe de la " prise " valant " commencement ", comme dans l'interprétation heideggérienne de l'Anfang (terme signifiant * commencement * et forgé à partir de fangen, prendre, saisir, attraper), mais sous le signe de la déprise, de l'écart : " Se séparer, se disjoindre de l'objet et de soi, se déprendre du pareil au même, mesurer sans cesse l'écart entre la chose possédée et le mot qui la désigne et qui, la désignant, dit d'abord qu'elle n'est pas là " (Pontalis, 1988, p. 193). La profération serait-elle ainsi dépourvue de toute forme de convocation? Pour être capable d'une telle ascèse, la parole devrait avoir totalement rompu avec cette dimension d'incantation que Marcel Détienne impute à sa forme première, celle qu'articulaient les " maîtres de vérité " (Détienne, 1973), avant qu'advienne la parole polémique, prenant naissance dans le cercle des guerriers. À cette parole qui ferait d'emblée accéder à l'existence ce qu'elle profêre, J.-B. Pontalis oppose une parole trouvant son essence dans une 
opération de dégagement, d'éloignement : Car le langage n'est pas prise : il ne saisit rien de la substance du réel, pas même le moindre prélèvement * (Pontalis, 1988, p. 193). L'insistance sur les tournures négatives a-t-elle elle-même pour fonction de conjurer quelque tentation romantique? Cette option en faveur d'une stratégie de désenchantement obligé n'empêchera pas, néanmoins, que soient entendues les protestations émises par ce qui est stigmatisé comme l'« appétit » du langage :

Puisqu' il est né de la perte et qu'il n'a rien qui soit à lui, son appétit est énorme! ll peut, il doit, pour vivre, tout * incorporer * jusqu'au corps et plus que lui; il séduit mieux qu'un sexe, [...] il a tous les pouvoirs. Dans ce mouvement qui le porte de la maittrise, de la magie, à la conscience de sa vacuité essentielle, il va osciller entre le triomphe maniaque et la mélancolie. Mais la mélancolie révèle sa nature, la manie seulement son effort. (Pontalis, 1988, pp. 194-195)

Réaffleure dans ces lignes la mélancolie du prince nordique - "Words, words, words ", disait Hamlet - auquel Freud oppose la réplique faustienne : Ne méprisons pas la parole. "L'efficacité de la parole dans le champ psychanalytique s'étayera en effet sur la dimension magique du langage autant ou plus que sur ses vertus ascétiques. N'assistons-nous pas ici à l'équivalent d'une guerre de religions, l'allusion au texte sacré se trouvant d'ailleurs discrètement insérée dans la proposition de Pontalis parlant de * la mauvaise nouvelle que porte un langage sans fin ni finalité, qui ne se propose ni l'expression, ni la communication. " " Ce sont là, ajoute-t-il, ses retombées, non sa raison d'être " $(1988$, p. 195). En se faisant anti-évangélique, en prescrivant le désenchantement systématique, la psychanalyse ainsi conçue renoue avec une antique discipline, celle que promeut Socrate, personnage de Platon dans Gorgias : déclarer la guerre à la « flatterie ", à tout ce qui risque de séduire et de caresser le désir, comme si le chemin de la vérité était reconnaissable aux égratignures qu'il inflige. Dans le conflit qui, dans Gorgias, oppose le cuisinier et le chirurgien - un chirurgien qui va d'ailleurs servir de modèle à la leçon promue par J.-B. Pontalis (1988, p. 197) -, il faut choisir celui qui, pour le plus grand bien du patient, va recourir aux instruments permettant de * couper et brôler ». À cette apologie de la lutte déclarée contre toute séduction (Schneider, 1994), Nietzsche opposera une stratégie capable de déjouer l'alternative vérité-illusion. 
Le traducteur va ainsi apparaître, dans la démarche de J.-B. Pontalis, sous les traits du chirurgien de la langue : * Un traducteur opère [...]. On dit que la qualité maîtresse du chirurgien est, à chaque instant, la décision " (1988, pp. 197-198). Confronté à une tâche non exempte de cruauté, il se présentera néanmoins en victime du devoir : * Le traducteur, je le vois d'abord comme un être en souffrance : il a perdu sa langue sans en gagner une autre »; et, plus loin : « Le traducteur doit être doué d'une capacité infinie d'être triste : il n'a pas le droit de jouer avec ses mots à lui, il n'a pas le pouvoir de restituer les mots de l'autre " (1988, pp. 197-198). Pour que soit mise en avant une telle déclaration d'impuissance, étayée sur un devoir de non-jouissance, ne faut-il pas supposer, en deçà de ces conduites d'excuse, la peur et l'attrait d'une transgression irrémédiable? Transgresser, traduire : deux verbes qui renvoient à une opération de passage.

\section{Le franchissement}

Aux apôtres dư désenchantement une concession sera faite en ce qui concerne le travail de traduction. $\mathrm{Si}$, en mettant entre parenthèses l'opération de passage, on isole le texte apparaissant au terme du travail, il est sans doute difficile d'idolâtrer le produit obtenu. Il en va tout autrement si on envisage la traduction, non dans ses produits finis, mais dans son exercice même. Exercice qui va d'ailleurs être interrogé ici dans sa phase rétroactive, celle qui consiste à confronter texte original et texte officiellement traduit. Il est vrai que cette position seconde, la seule dont je puisse parler par expérience, est beaucoup moins ingrate que celle qui revient au traducteur de première ligne.

Avouons qu'il est difficile de mettre au compte de la mélancolie l'humeur qui porte le mouvement de retour aux textes considérés comme fondateurs. Retour que j'ai da pratiquer en cernant, dans Freud et le plaisir et dans Don Juan et le procès de séduction, la place de la séduction dans l'ouvre de Freud et en débridant les lèvres d'une blessure d'enfance dans * Père, ne vois-tu pas...? *. Comment d'ailleurs éviter le piège mégalomaniaque, lorsqu'il s'agit d'arracher aux mains des infidèles - et les traducteurs avouent ou revendiquent ce statut! - les écrits instaurateurs d'une autre logique, d'un autre regard? Il est vrai que la 
confrontation entre les textes de Freud et les premières traductions est riche d'effets de surprise, surtout si on s'attache aux premières explorations.

La condamnation de la séduction, qui préside à la décision de placer le recours au langage sous le signe de la mélancolie, est certes agissante dans les décisions prises par les premières traductions officielles. Dans les lettres à Fliess à l'occasion desquelles Freud livre les expériences qui sous-tendent l'un de ses premiers revirements théoriques - abandon déclaré de la " neurotica " et recours au mythe odipien -, un souvenir a une portée décisive : la découverte par Freud, dans sa propre histoire, d'une expérience de séduction. Séduction perpétrée, non par le père, comme les hypothèses cliniques le donnaient à penser, mais par la femme qui fut la nourrice. Or contrairement au mouvement accusateur qui s'adressait aux pères séducteurs, on assiste, vis-à-vis de cette séductrice, à des déclarations empreintes de lyrisme : « Je garderai à la vieille femme un souvenir reconnaissant pour m'avoir donné, à une époque aussi précoce de ma vie, les moyens de vivre et de continuer a vivre * ([lettre du 04-10-1897], 1956, p. 194; 1986, p. 289). L'initiatrice reçoit ainsi le titre de * professeur en choses sexuelles * et Freud la désigne * meine Urheberin ". Urheber renvoie à l'artisan et il est difficile de traduire ce terme en français. "Génératrice " fut le terme choisi, correspondant d'ailleurs à la décision prise par le traducteur anglais, James Strachey. Mais, estimant sans doute que cette louange ainsi adressée à la séductrice était inconvenant, Strachey a ajouté, à la suite de " generator * et entre crochets : " [of neurosis] ". Décision qui casse le mouvement freudien de reconnaissance; selon Freud, cette femme lui a donné l'équivalent d'une seconde vie, selon Strachey, elle n'a fait que lui octroyer une névrose. Les traducteurs d'autres pays emboitent le pas au traducteur rectificateur et, en français comme dans les langues hispaniques, on retrouve mentionné, ajouté entre crochets, le don de la névrose. Les héritiers font ainsi intrusion dans l'enfance de Freud pour mettre au pilori ce personnage féminin.

Condamnation de la femme séductrice ou condamnation de la " chose sexuelle $n$ ? La défiguration vertueuse se poursuit au moment où Freud évoque son rapport actuel à la sexualité. Il livre alors cet aveu que je transcrirai d'abord dans la traduction française : * Une personne comme moi n'a plus que faire de l'excitation sexuelle, mais je reste pourtant 
serein "([lettre du 30-10-1897], 1956, p. 201; 1986, p. 298). Le recours au texte, s'agissant de la seconde proposition, oriente vers un aveu quelque peu différent : "Ich bin aber noch immer freudig dabei ", ce qui peut être rendu par " mais j'y trouve toujours du plaisir " ou, si l'on veut serrer le texte de plus près : * mais cela me rend toujours joyeux *. Le terme de freudig est loin d'être indifférent, puisqu'il renvoie au patronyme de Freud. Cette déclaration certes ambiguě, puisque Freud avoue à la fois son retrait et sa participation joyeuse, se trouve ainsi assagie, voire bâillonnée, par les traducteurs français. S'agit-il du retour obstiné du manteau de Noé? À la condamnation de la séductrice il faut donc ajouter la construction d'une fiction faisant refluer dans l'ombre le maître jouisseur, cette ombre dont Don Juan ne sort que pour y retourner à la fin de la pièce, afin que soit campée une noble figure de sage, arborant un masque * serein ". L'institution psychanalytique aurait-elle besoin de poser, au départ de l'itinéraire et afin que soit conjurée la tentation émanant de quelque séductrice, un maître ininflammable?

Loin de faire tomber sur le texte freudien un flou généralisé, il semble que la traduction française ait ciblé étroitement les points qu'il importait d'occulter, en imposant parfois au texte de sérieux remaniements. Lors du passage où Freud, dans $L$ Interprétation des rêves, relate le rêve de la prothèse éjectée, rêve livré par une patiente, un curieux changement intervient dans le passage de lallemand au français. Le contexte est ainsi campé par Freud :

Une de mes malades, qui avait dô subir une opération à la mâchoire, opération qui avait mal réussi, devait, selon le désir des médecins, porter jour et nuit au niveau de sa joue malade un appareil reffigerant. Mais elle avait l'habitude de l'arracher dès qu'elle était endormie. Un jour on me pria de lui adresser des reproches à ce sujet; elle avait de nouveau jeté son appareil sur le sol. La malade se justifia : « Cette fois, je n'y peux vraiment rien; c'était l'effet d'un rêve que j'ai eu cette nuit. J'êtais en rêve dans une loge à l'Opéra et je m'intéressais vivement à la représentation. Mais, au sanatorium, M. Karl Meyer était couché et gémissait atrocement à cause de douleurs à la mâchoire. J'ai dit que, puisque moi je ne souffrais pas, je n'avais done pas besoin d'appareil; c'est pourquoi je l'ai jeté au loin. * $(1967$, p. 116; 1972, p. 144)

La traduction ici proposée est extraite du texte que j'ai consacré à L'Interprétation des rêves, « Père, ne vois-tu pas...? * (1985), à l'occasion 
duquel j'ai retraduit un certain nombre de passages. La logique qui soustend le rêve n'est en effet accessible que si on respecte l'identité de la zone corporelle souffrante : après l'« opération à la mâchoire n et les douleurs qui s'ensuivent, la malade ejjecte cette souffrance en direction d'un autre personnage, $M$. Karl Meyer, qui hérite en rêve de ces a douleurs à la mâchoire ", ce qui permet l'affirmation : a moi je ne souffrais pas *. Or la traduction Meyerson-Berger procède a un changement de zone en affirmant : « $\hat{A}$ la clinique, il y avait M. Karl Meyer qui se plaignait de terribles maux de tête ", ce qui rend inintelligible le processus de transfert et d'identification projective. Pourquoi avoir maquillé le Kieferschmerzen (douleurs à la mâchoire), alors que le terme de * mâchoire " est bien présent dans la description de la situation qui préside au rêve; est effectivement mentionnée l' $\alpha$ opération à la mâchoire " (Kieferoperation). Mais n'est-ce pas précisément ce surgissement inopiné d'une * opération à la mâchoire " qui a alerté les héritiers traducteurs? Il est en effet difficile de ne pas songer aux multiples opérations à la mâchoire que connut Freud du fait de son cancer, ce qui confère à ce récit précoce une dimension prémonitoire, en quelque sorte oraculaire. Le thème de l'éjection, en direction d'un autre, d'une bouche malade est d'autant plus saisissant que cette figure qui hante L'Interprétation des rêves - bouche d'Irma, bouche de cette patiente, bouche du fils officier - renvoie précisément à la blessure d'enfance que connut Freud, dont il fait état à la suite du rêve du fils officier. Or cette blessure fit probablement retour dans le réel, lorsque Freud confia au bistouri de Fliess la mâchoire d'Emma Eckstein et que s'ensuivirent des complications infectieuses et hémorragiques. Freud aurait-il tenté de transférer à sa patiente ce qui faisait pour lui l'objet d'un souvenir traumatique, souvenir éclaté et errant? Le rêve livré par la patiente prend alors une dimension vertigineuse, comme si cette dernière avait elle-même rêvé, en une sorte d'avant-coup prémonitoire, cette stratégie visant à transférer sur l'autre, à éjecter en direction de l'autre, une douleur insoutenable.

Les traducteurs ont-ils tenté de s'interposer pour arrêter le circuit infernal de la douleur, ses effets de boomerang, en faisant disparaître les " douleurs à la mâchoire " pour leur substituer des symptômes moins inquiétants, ces " terribles maux de tête ", qui présentent le mérite de mieux convenir au personnage magistral que fut Freud? Voiler la jouissance sexuelle du maître, faire disparaître magiquement ses zones 
vulnérables : ce qui, du maître, doit être occulté correspond précisément à ce qui fait de lui un être exposé.

Le transfert de la vulnérabilité et de l'exposition à l'autre se poursuivra d'ailleurs dans le travail théorique. C'est à loccasion des études portant sur " La féminité " et sur " La sexualité féminine " que Freud revient explicitement sur la possibilité d'une séduction advenue dans le réel, la séduction liée aux soins maternels étant d'ailleurs nécessairement vécue par la petite fille. Par la petite fille et non par le petit garçon? Curieusement, sans mettre officiellement à l'abri le petit garçon par rapport à l'efficience attachée aux mains maternelles, Freud se contente de le protéger par la magie du langage, en le tenant à l'écart d'une nomination qui le désignerait. Dans ses analyses du plaisir sexuel, à l'occasion des Trois Essais, la peau exposée à la séduction venant de l'autre ne recouvrira que le corps féminin : peau des seins (Brusthaut), peau du vagin, la participation masculine se limitant essentiellement au plan moteur. Les évitements des héritiers traducteurs, campant un maître protégé contre la vulnérablité et contre l'exposition à la séduction, prolongent ainsi certains axes structurant l'approche théorique.

Le travail d'atténuation et d'occultation peut d'ailleurs s'exercer insidieusement à l'occasion de thèmes qui ne mettent pas en jeu le partage sexué. Se penchant précisément sur la question de l'atténuation et de l'assourdissement des affects à l'intérieur même du travail du rêve, Freud propose une comparaison permettant de prendre la mesure de l'écart se creusant entre l'intensité et la violence des souvenirs situés à la source du rêve et l'étouffement auquel se livre l'atelier de fabrication du rêve. Or ce même étouffement va faire l'objet, dans la traduction, d'une mise en abyme, puisque la courte proposition - * Cest la paix après la bataille $n-$, proposition qui ponctue le développement sur l'atténuation des affects, vient se substituer á une évocation plus accentuée : « C'est comme la tranquillité d'un champ de cadavres (die Ruhe eines Leichenfeldes); on ne ressent plus rien de la fureur (Toben) du combat " $\left(1967\right.$, p. $298 ; 1972$, p. $\left.450^{1}\right)$. Après le passage des héritiers traducteurs, se trouve nettoyé le * champ de cadavres *, aboli le Toben (déchaînement de fureur) du combat. Pourquoi avoir ainsi censuré une version hard pour

' Ce passage a été analysé dans “ Père, ne vois-tu pas...? », p. 202. 
lui substituer une version soft, et ceci précisément au moment où Freud montre la difficulté, dans le travail analytique, d'aller à contre-courant de l'atténuation et de l'insonorisation qui peuvent être à l'œuvre dans le rêve?

En bonne logique psychanalytique, toute proposition est réversible, et je pourrais faire porter le soupçon sur ce qui m'attire dans la restitution d'une version hard. Un plaidoyer indirect pourrait être tenté. René Kaës prend l'exemple du masquage de la violence dans l'opération pratiquée par Fliess sur Emma Eckstein, masquage soutenu par la décision freudienne de disculper Fliess, et avance la notion de « pacte dénégatif " pour rendre compte de tout ce qui doit se trouver dénié lors de la constitution d'un groupe ou la mise en place d'une institution : * Que le maintien du lien soit exigé au prix du négatif par ses sujets mêmes, écrit René Kaës, aucune autre figure que celle de l'alliance sanglante de Freud, d'Emma Eckstein et de Fliess ne saurait mieux, pour des psychanalystes, la représenter, puisqu'elle se place à l'origine de la psychanalyse " $(1989$, p. 110). Le déni trouvera son centre de gravité dans la volonté, propre à Freud, de protéger Fliess contre toute responsabilité dans le sang versé : * En disculpant Fliess ("Pour ce qui est du sang, tu n'es absolument pas coupable"), poursuit René Kaës, il fait "porter la créance du sang d'Emma à l'hystérie de celle-ci" (Philippe Réfabert, Barbro Sylwan) * (1989, p. 110). René Kaès dégage ainsi la portée de ce déni fondateur :

Cet épisode iliustre, au cceur du débat sur la séduction, le pacte dénégatif conclu, à leur insu, entre Freud et Fliess à propos d'Emma Emma est ici la figure, pour ces deux hommes, du trou qu'ils veulent explorer et réduire en lui donnant un contenu de gaze et de sang. Leur pacte est à la fois la dénégation de ce désir, le déni de leur lien homosexuel fondé sur l'effacement de l'inconnu de la féminité [...]. Instituer la psychanalyse, c'est placer au coeur de son débat le proton pseudos et la question de la vérité du sujet dans son rapport à ce qui le représente : pour Freud, Fliess autant qu'Emma. (1989, p. 110)

Or le travail de traduction, lorsqu'il se trouve pris dans ces enjeux institutionnels, peut s'inscrire dans le sillage d'un tel pacte dénégatif. II s'agira de renvoyer au dehors un régime de prédation qui sera considéré comme étranger à l'esprit du groupe ou de l'institution. Blanchir, gommer deviennent alors des mesures protectrices venant soutenir le narcissisme du groupe, ce qui permet d'insonoriser la violence qui peut se jouer à l'intérieur de l'enceinte. Dans une telle perspective, les opérations prenant 
place dans le champ psychanalytique sont nécessairement duplices. Impossible d'échapper radicalement à ce " pacte dénégatif $"$; d'où le mouvement de reflux qui, s'emparant de l'auto-analyse, est amené à se heurter au proton pseudos. Mensonge inaugural, nucléaire, dont les ramifications pourront être approchées, ou supputées, si on est attentif au travail de la nomination dans sa double face d'occultation et de fondation. Or la traduction et la retraduction s'attachent précisément à ce qui se trame aux frontières de tout champ, à tout ce qui touche aux précautions conjuratoires visant à rendre intouchables certaines figures d'autorité, celle du maître en l'occurrence, figures exerçant un pouvoir paradigmatique sur la configuration des territoires sexués.

\section{L'inouĩ de l'autre langue}

Est-il néanmoins pertinent de supposer une intention de masquage ou une opération dénégative là où le passage d'une langue à l'autre semble buter sur un écueil? Éprouver la résistance, la non-convertibilité de l'autre langue peut correspondre à une expérience où interfèrent aussi bien le sentiment d'impuissance que le triomphe. Prendre en flagrant délit l'opération de masquage ne peut que donner le sentiment d'être sur le seuil d'un secret inviolable. Il n'est d'ailleurs pas nécessaire, pour accéder à cette saisie de l'inviolable, de se trouver en présence de pratiques visant l'occultation délibérée, comme dans l'invention dérisoire des * terribles maux de tête ". La rencontre de signifiants difficilement traduisibles, quelle que soit la bonne volonté des traducteurs, peut conduire a une sacralisation de ces termes qui semblent défier la loi de substitution indéfinie qui semble être celle du langage. Les mots sont alors pris dans un perpétuel renvoi à eux-mêmes, comme si la signification devenait inséparable de la profération. L'exhumation par Lacan de das Ding remplit une telle fonction; devant l'épreuve de l'intraduisible, s'entrevoit la différence, typique de l'entrée dans le sacré, entre les initiés et les autres. De tels vocables semblent résister à la loi de l'échange, comme s'ils emportaient avec eux les réseaux de significations qui se sont tissés dans une langue singulière. N'est-on pas alors en présence de l'opération inverse de celle que Greimas a rendue solidaire de la circulation usuelle de la plupart des mots, la " désémantisation n? Dans la banalisation de l'échange, les termes courent en effet le risque d'une démonétisation. L'épreuve de la traduction, lorsqu'elle se heurte au difficilement traduisible, provoque alors ce qu'on pourrait nommer une 
sursémantisation, surinvestissement réservé à ceux qui, familiers de ce qui circule de chaque côté de la frontière, deviennent alors les témoins privilégiés de saveurs sémantiques réservées à la jouissance privée de quelques palais polyglottes. Les autres devront se contenter de la seule profération.

Dans la tentative répétée pour capter la teneur signifiante d'un terme, les essais de traduction vont s'acharner, en des mouvements de flux et de reflux, à rendre sensibles les écarts repérables sur un double registre : écarts entre les signifiants d'une même langue - selon la loi de négation réciproque et de différenciation que fait affleurer Ferdinand de Saussure -, écarts entre ces mêmes termes et leurs traductions éventuelles. N'assiste-t-on pas alors à l'équivalent de quelque multiplication des pains? Certes, aucun ne convient absolument, ce qui met à mal la passion de l'adéquation, mais on n'en assiste pas moins à un jeu avec l'abondance. Je tenterai de rendre compte de cet enchevêtrement de réseaux à partir d'un signifiant allemand difficilement repérable dans les traductions françaises de l'œuvre freudienne, le terme de Leib.

Un seul vocable français, " corps", pour rendre deux termes que distingue la langue allemande, Leib et Körper. Le premier de ces termes a d'ailleurs posé des problèmes aux phénoménologues, en particulier aux traducteurs et commentateurs de Husserl, qui ont mis l'accent sur la notion de " corps vivant ", terme d'ailleurs mis en concurrence avec celui de " chair $n$. Or le rapport au vivant est suggéré par l'étymologie elle-même, qui fait dériver Leib de leben (vivre). Leib renverrait ainsi au corps vécu, animé, approché dans son réseau de mouvements internes, alors que $K o ̈ r p e r$, bien que pouvant désigner lui aussi le corps humain, couvre le registre de l'ensemble des corps du monde physique. Chaque désignation devient ainsi porteuse d'une philosophie implicite, puisque tout corps, physique ou humain, relève, dans l'optique cartésienne, de la catégorie du Körper, alors que la perspective phénoménologique donne vie, pourraiton dire, au Leib.

La grille fournie par les diverses perspectives philosophiques estelle de quelque secours pour éclairer l'apparition de Leib et de Körper dans le texte freudien? Sans que soit exclue la possibilité d'une investigation s'engageant dans cette voie, un premier niveau de repérage nous engage dans une piste assez différente. Dans l'écriture freudienne, 
une surimpression s'impose entre la distinction Leib-Körper et celle qui commande la différence sexuelle. Le Leib n'apparaît en effet, dans la plupart des occurrences, que dans le voisinage du féminin, envisagé essentiellement dans sa potentialité maternelle. Les hommes seraient-ils donc dépourvus de Leib et réduits au statut corrrespondant à celui de la charpente et de l'ossature, les termes d' $\alpha$ armature » et de $~$ carcasse » étant offerts par le dictionnaire Bertaux-Lepointe comme sens possibles de Körper? Une théorie sexuelle implicite serait-elle agissante au niveau même de la langue, théorie sur laquelle buterait l'aventure de la traduction?

Il est vrai que, dans le mouvement des textes freudiens, la corrélation entre le Leib et le féminin n'est efficiente que lorsqu'il est question de s'interroger sur les rapports que la femme entretient avec son corps. Une telle corrélation est indirecte : c'est dans la pensée de l'enfant, en particulier lorsque ce demier s'interroge sur son origine, que le corps féminin est désigné comme Leib, terme d'ailleurs inséré dans une formule plus large : im Leibe der Mutter (dans le corps de la mère). Ce qui est dit de ce corps concerne moins son apparence, sa Gestalt, que son pouvoir d'abriter quelque hôte à l'intérieur de lui. Le Leib semble donc essentiellement un corps en creux. Le texte de Freud sur * Les théories sexuelles infantiles » (1908) fait d'ailleurs voisiner, à l'intérieur même de l'espace scripturaire, la mention du Leib comme premier lieu de séjour de l'enfant, un enfant qui " croît dans le corps de la mère (im Leibe der Mutter) " et la présentation de l'espace réservé à l'arrivée du père en elle, l'« espace creux (Hohlraum) " $(1969$, p. 21 ; G.W., VII, p. 180), espace dont l'enfant ne prendra connaissance que plus tard.

Dans le paragraphe qui précède l'apparition du creux maternel, le regard est porté sur les privilèges attenant au corps masculin. La petite fille, note Freud, " développe un grand intérêt pour cette partie du corps du petit garçon " $(1969$, p. $21 ; G . W$. , VII, p. 180). Le terme utilisé pour désigner le corps n'est plus alors $L e i b$, mais Körper; l'estime s'adresse au Körperteil (partie du corps). À partir du Körper, s'organise le mouvement qui s'oriente vers l'extériorité et qui se manifeste dans la motricité. Dans "Pour introduire le narcissisme ", la comparaison entre le moi et la stratégie attribuée aux * animalcules ", comme l'amibe, fait intervenir le Körper comme base de lancement des pseudopodes ; $\propto[. .$.$] l'investissement$ 
du moi [...] se comporte comme le corps (Körper) d'un animalcule protoplasmique envers les pseudopodes qu'il a émis n $(1969$, p. 83; G.W., X, p. 141).

Leib et Körper s'organisent ainsi autour d'un renversement de vecteur : orientation soit vers l'intérieur, soit vers l'extérieur. Comment d'ailleurs limiter et déterminer cet espace interne attribué à l'enfant comme premier habitacle plus qu'à la femme? Si l'indication d'un creux s'offrant à être rempli semble faire intrinsèquement partie de la détermination du Leib, les traductions divergent quant à l'extension et à la localisation précise d'un tel creux. Traducteur des * Théories sexuelles infantiles ", J.B. Pontalis, dans les passages précédemment cités, recourt à l'expression " corps de la mère " - choix qui semble le plus légitime - pour traduire * Leib der Mutter n. Jean Laplanche, quant à lui, responsable du texte français de "L'organisation génitale infantile ", opère un autre choix : * L'enfant vit dans le ventre (intestin) de la mère (im Leib (Darme) der Mutter) et est mis au monde par l'orifice intestinal $~(1969$, p. $116 ; G . W$., XIII, p. 297). Le thème de la naissance intestinale correspond certes à une théorie sexuelle infantile très fréquente, mais en identifiant d'emblée le $L e i b$ et le " ventre " - traduction certes possible, bien que l'équivalence entre Leib et ventre ne corresponde qu'à un champ de possibilités restreintes -, on ferme prématurément la question posée par l'enfant masculin, question qui traverse toute la thematique de l'Homme aux loups. Dans la traduction de Luc Weibel, relue par J.-B. Pontalis et Cornélius Heim, après l'évocation des " enfants retirés du corps (Leib) du loup ", la question est ainsi posée : " Le loup était-il alors un être féminin ou des hommes pouvaient-ils également avoir des enfants dans leur corps (im Leib)? "

La prospection en direction des pouvoirs dévolus à l'intestin peut certes se mettre en place à la suite de cette question, mais il n'est pas certain que cette réponse suffise à apaiser l'angoisse de l'enfant, dans la mesure où s'entrevoit aussi l'éventualité d'un privilège féminin, ce qui conduit à organiser la différence sexuelle autour d'un autre manque que celui qui concerne la présence ou l'absence de pénis. Seule la traduction de Leib par " corps " rend possible l'ouverture de la question, dans la mesure où l'espace interne, s'ouvrant dans le corps féminin, peut conduire à l'hypothèse d'un * espace creux " qui ne corresponde pas nécessairement, encore que cette assignation ne soit pas interdite, au lieu intestinal. La 
traduction de Leib par " corps * laisse planer une indétermination plus grande que lorsque le terme choisi est " ventre ". Indétermination qui permettra d'ailleurs que, dans la traduction de "L'inquiétante étrangeté ", Bertrand Féron ait choisi de rendre Leib par \& sein *, terme d'ailleurs lourd de connotations religieuses plus qu'anatomiques. La question, en l'occurrence, n'est pas celle du corps envisagé dans la séparation, puisque l'effet d'« inquiétante étrangeté » est rapporté par Freud à l'appréhension provoquée, chez certains hommes, par la vue du sexe féminin, en tant que corrélé à un processus de filiation. Loin de se référer, en effet, à une localisation circonscrite, un tel lieu renverrait à l'origine de tout être : « Il se trouve que cet étrangement inquiétant est l'entrée de l'antique terre natale (der Eingang zur alten Heimat) du petit d'homme [...]. Quand le rêveur pense, jusque dans le rêve, à propos d'un lieu ou d'un paysage : "Cela m'est bien connu, j'y ai déjà été une fois", l'interprétation est autorisée à y substituer le sexe ou le sein (Leib) de la mère " (1989, p. 252; G.W., XII, p. 259). Dans une telle perspective, le lieu de séjour originaire s'inscrit moins dans la trajectoire déployée par la mère que dans celle qui appartient à l'enfant; si le Leib maternel est notre première " patrie ", Heimat - terme qui, renvoyant à l'intimité qui s'annonce dans le heim, désigne un habitacle apprivoisé, situé à l'abri des déjections solidaires de la représentation cloacale du " ventre " -, un tel Leib se rapporte d'ailleurs moins à la mère qu'à l'enveloppement cutané, au " moipeau » qui porterait le corps de l'enfant. La question du Leib, chez Freud, est régulièrement corrélée à l'interrogation sur la possibilité de gestation qui serait attribuée ou refusée à l'être masculin.

La menace d'évidement charnel qui est solidaire de l'inscription du masculin du côté du Körper et non du Leib fait d'ailleurs l'objet d'une figuration suggestive dans un texte de Saint-Denys Garneau, cité par Anne Élaine Cliche :

Cette idée des os consistait à se dépouiller de la chair à laquelle on ne peut jamais se fier [...]. Maintenant, c'est l'idée de l'épine dorsale avec cette impression en plus d'une hache qui (sans douleur) en détache les côtes, l'impression d'être ébranché [...]. Maintenant il sera réduit à ce seul tronc vertical, franchement nu. (1995, pp. 95-96) 
Se profile ainsi une charpente qui correspond étonnamment aux diagrammes du masculin, tels qu'ils apparaissent dans l'œuvre de Françoise Héritier ou dans celle de Nicole Loraux : accès à une verticalité décharnée.

$\mathrm{Si}$, dans le partage Leib-Körper, Freud refuse à l'homme, par le truchement du lexique auquel il a recours, l'accès à une corporéité vivante, du moins lui offre-t-il, par la grâce du jeu analytique, une participation métaphorique à ce Leib refusé à sa masculinité. Le lieu à l'intérieur duquel opère l'inconscient se trouve en effet figuré, quel que soit le sexe de l'analysant, par l'intériorité corporelle féminine :

Si a l'inconscient ", en tant qu'élément de la pensée du rêve, doit être figuré dans le têve, il est remplacé (ersetzt), de manière tout à fait adéquate, par des localités « souterraines n qui, dans d'autres occasions, sans aucun rapport avec la cure analytique, avaient signifié le corps de la femme ou celui de la mère (den Frauenleib oder den Mutterleib). (Freud. 1967, p. 351 [traduction modifiée]; Freud, 1972, p. 399).

Tant que dure le temps d'une analyse, l'être masculin serait donc amené, s'il veut s'intéresser à son inconscient, à opérer l'équivalent d'un changement de sexe : le Leib qui sert de logis métaphorique à l'inconscient entretient en effet un rapport de prédilection, dans le lexique allemand, avec le monde des femmes ou des mères.

\section{CNRS et Paris VII}

\section{Références}

CLICHE, Anne Élaine (1995). Comédies. L'Autre Scène de l'écriture. Montréal, XYZ éditeur.

DÉTIENNE, Marcel (1973). Les maîtres de vérité dans la Grèce archaïque. Paris, Maspero.

FREUD, Sigmund (1941). « Uber infantile Sexualtheorien ". Gesammelte Werke, VII. Frankfurt am Main, S. Fischer Verlag, pp. 171-188.

- (1946). "Zur Einführung des Narcissmus ". Gesammelte Werke, X. Frankfurt am Main, S. Fischer Verlag, pp. 137-170. 
- (1947). * Das Unheimliche *. Gesammelte Werke, XII. Frankfurt am Main, S. Fischer Verlag, pp. 229-268.

- (1950). * Die infantile Genitalorganisation *. Gesammelte Werke, XIII. Frankfurt am Main, S. Fischer Verlag, p. 293-298.

- (1956). Naissance de la psychanalyse. Paris, Presses Universitaires de France.

- (1967). L'Interprétation des rêves. Paris, Presses Universitaires de France.

- (1969). "Les théories sexuelles infantiles ". Dans La Vie sexuelle. Paris, Presses Universitaires de France, pp. 14-27.

- (1969). "Pour introduire le narcissisme ". Dans La Vie sexuelle. Paris, Presses Universitaires de France, pp. 81-105.

- (1969). "L'organisation génitale infantile ». Dans La Vie sexuelle. Paris, Presses Universitaires de France, pp. 113-122.

-(1972). Die Traumdeutung. Frankfurt-am-Main, S. Fischer Verlag, coll. "Studienausgabe".

- (1986). Briefe an Wilhelm Fliess. Frankfurt am Main, S. Fischer Verlag.

- (1989). L'Inquiétante Étrangeté et autres essais. Paris, Gallimard.

HÉRITIER, Françoise (1996). Masculin/Féminin, Paris, Éd. Odile Jacob

KAËS, René (1989). « Le pacte dénégatif dans les ensembles transsubjectifs ", dans Le Négatif. Figures et modalités. Paris, Dunod, pp. 111-136.

LORAUX, Nicole (1989). Les expériences de Tirésias. Paris, Gallimard, coll." NRF/Essai ".

PONTALIS, J.- B. (1988). Perdre de vue. Paris, Gallimard. 
SCHNEIDER, Monique (1980). La Parole et l'inceste. Paris, AubierMontaigne, 1980.

- (1985). "Père, ne vois-tu pas...? * Le Père, le maître, le spectre dans L'Interprétation des rêves. Paris, Denoël.

- (1994). Don Juan et le procès de la séduction. Paris, Aubier.

RÉSUMÉ : Éprouver le passage - Lorsque se trouve accentuée la dimension de perte inhérente à l'accès au symbolique, la traduction peut apparaître comme le champ privilégié où s'étale cette perte. Direction suivie par J.-B. Pontalis parlant de " la mélancolie du langage " et dotant le traducteur de " la capacité infinie d'être triste ". Une autre orientation se dessine si la traduction est envisagée dans son exercice même et non dans son seul résultat. L'expérience analysée sera celle de la retraduction des textes de Freud, mettant au jour les opérations à l'œuvre dans les traductions officielles : maquillage des termes se rapportant à la scène de séduction et à la possibilité, pour le maître, d'avoir accès à la jouissance. Ce qui conduit à une mise en abyme du procès de la séduction.

De la traduction mystifiée a la traduction impossible : c'est la tentative même de franchissement qui fait apparaître l'inconvertible dans une langue, l'exemple choisi étant le signifiant allemand, Leib, terme désignant, par opposition à Körper, le corps vivant ou le corps en creux, donc connoté de féminité; connotation qu'aucun terme français ne pourra rendre.

ABSTRAC' : Experiencing the Passage - When examining the loss involved in the attempt to accede to the symbolic, translation can appear to be a privileged field in that it is there that the loss occurs. This line of thought is pursued by J.-B. Pontalis, who talks about "the melancholy of language" and endows translators with an "infinite capacity for sadness." Another view of translating comes from placing the emphasis on the process of translating rather than on the translation as product. The author analyzes the retranslation of Freud's writings, revealing the operations at work in the official translations : the disguising of terms referring to the seduction scene and to the possibility, for the master, of experiencing jouissance. This leads to the mise en abyme of the seduction process.

From mystifying translation to impossible translation : the very attempt to cross over reveals the inconvertible in a language; for example, 
the German signifier, Leib, a term designating, in opposition to Korper, the living or receptive body, has a connotation of femininity no French term can render.

Monique Schneider : 19, rue Bobillot, app. A-260, 75013 Paris, France. 